\title{
Expression and Clinical Significance of Sushi Domain- Containing Protein 3 (SUSD3) and Insulin-like Growth Factor-I Receptor (IGF-IR) in Breast Cancer
}

\author{
Shuang Zhao ${ }^{1,2 \&}$, Shuang-Shuang Chen ${ }^{1,3 \&}$, Yuan Gu ${ }^{4 \&}$, En-Ze Jiang ${ }^{1,2}$, Zheng- \\ Hong Yu ${ }^{1,2 *}$
}

\begin{abstract}
Background: To investigate the expression of insulin-like growth factor-I receptor (IGF-IR) and sushi domaincontaining protein 3 (SUSD3) in breast cancer tissue, and analyze their relationship with clinical parameters and the correlation betweenthe two proteins. Materials and Methods: The expression of IGF-IR and SUSD3 in 100 cases of breast cancer tissues and adjacent normal breast tissues after surgery was detected by immunohistochemical technique MaxVisionTM, and the relationship with clinical pathological features was further analyzed. Results: The positive rate of IGF-IR protein was $86.0 \%$ in breast cancer, higher than 3.0\% in adjacent normalbreast tissue $(P<\mathbf{0 . 0 5})$. The positive expression rate of SUSD3 protein was $78.0 \%$ in breast cancer, higher than $2.0 \%$ in adjacent normal breast tissue $(P<0.05)$. The expression of IGF-IR and SUSD3 was related to estrogen receptor and pathological types $(P<0.05)$, but not with age, stage, the expression of HER-2 and Ki-67 $(P>0.05)$. The expression of IGF-IR and SUSD3 in breast cancer tissue was positively related $(r=$ $0.553, P<0.01)$. Conclusions: The expression of IGF-IR and SUSD3 may be correlated to the occurrence and development of breast cancer. The combined detection of IGF-IR, SUSD3 and ER may play an important role in judging prognosis and guiding adjuvant therapy after surgery of breast cancer.
\end{abstract}

Keywords: cancer; Insulin-like growth factor-I receptor (IGF-IR); Sushi domain-containing protein 3 (SUSD3)

Asian Pac J Cancer Prev, 16 (18), 8633-8636

\section{Introduction}

Breast cancer, which is a serious threat to women's health, tends to occur at a younger age and has become the most common malignant tumor in urban women. Despite the increasing incidence of breast cancer, the mortality of breast cancer continues to decline in developed countries; while both incidence and mortality rate of breast cancer is rising in developing countries as a whole (So et al., 2013). One of the risk factors for breast cancer is estrogen accumulation and excessive estrogen exposure (Abbasi et al., 2013; Yu et al., 2013b). And aromatase inhibitors (AIs) are the most effective endocrine therapy for postmenopausal estrogen-dependent breast cancer (Bulun et al., 2012; Dabydeen et al., 2015). AIs resistance, however, is the most common cause of endocrine therapy failure.Breast cancer is a highly heterogeneous tumor,which means breast cancers with same clinical and pathological features may have different biological behaviors, different responses to treatment and different prognosis.The currently used biomarker of AIs response, namely estrogen receptor $\alpha /$ progesterone receptor (ER $\alpha$ / PR), can only predict half of potential response to AIs treatment. Thus, a new tool to predict response to aromatase inhibitors is required. Insulin-like growth factor receptor-I (IGF-IR), which is highly expressed in many malignant tumors, is a transmembrane tyrosine kinase receptor.IGF-IR is also demonstrated to have interaction with estrogen receptor (ER), while SUSD3 (sushi domaincontaining protein 3) is a new gene in E2 / ER pathway (Yu et al., 2013c). In the present study, we investigated the expression of IGF-IR and SUSD3 in breast cancer tissue, and analyzed their relationship with clinical parameters and the correlation between the two proteins.

\section{Materials and Methods}

\section{Patient selection}

The formalin-fixed paraffin-embedded tissue specimens were collected from the breast cancer tissues and their corresponding adjacent tissues of 100 patients who underwent breast surgery during January 2008 to December 2012 in Nanjing General Hospital. All patients were female, aged 25 to 82 , with a median age of 50,42 cases aged over 50 years, 58 cases aged 50 years and younger. All specimens were histologically confirmed.The 
biomarker expression of the investigated tissue samples was as follows: ER- $(n=25), E R+(n=25), E R++(n=25)$, ER+++ $(n=25)$; HER-2 - $(n=26)$, HER-2 + $(n=45)$, HER$2++\sim+++(n=29)$; Ki-67>50\% $(n=10)$; triple negative breast cancer $(n=10)$. Pathological types: invasive ductal carcinoma 90 cases, invasive lobular carcinoma 10 cases; 8 patients had a family history of breast cancer; 3 died during follow-up, including 1 case of triple negative breast cancer; 5 cases of recurrence and metastasis, including 1 case of triple negative breast cancer and 1 case of ER (-) PR (-) HER-2 (+) patient. Among 75 cases of ER-positive patients, 56 patients received endocrine therapy. HER-2 was detected by immunohistochemistry, according to "China breast cancer HER-2 detect guideline (2009 Edition)", non-stained cases were defined as negative. According to"TNM Classification of Malignant Tumours ( 7th edition )" by UICC and AJCC, 18 were stage I, 49 were stage II, 23 were stage III, 10 were stage IV.

\section{Reagents and instruments}

IGF-IR antibody was purchased from Wuhan Boster biotechnology company (rabbit polyclonal $1: 100$ ),SUSD3 antibody was purchased from Abnova Corporation (rabbit polyclonal $1: 200$ ). MaxVisionTM detection system was purchased from Fuzhou Maixin Biotech. Co.,Ltd.

\section{Immunohistochemistry}

Immunochemistry (IHC) was carried out with MaxVisionTM detection system to detect the expression of IGF-IR and SUSD3. All specimens were formalin-fixed and paraffin-embedded. Sections of 5- $\mu \mathrm{m}$ thickness were dewaxed with xylene and dehydrated through a graded alcohol series. Briefly, antigen retrieval was performed and the slides were allowed to cool at room temperature. Wash the slides in distilled $\mathrm{H}_{2} \mathrm{O}$.Wash the slides with PBS (pH 7.4) for $1 \mathrm{~min}$ and drain PBS with dry gauze. Add $50 \mu \mathrm{L} 3 \% \mathrm{H}_{2} \mathrm{O}_{2}$ solution on each slide to inhibit the endogenous peroxidase activity, and all slides were incubated at room temperature for $10 \mathrm{~min}$. Wash slides in PBS, 3 x 1 minute, and drain PBS with dry gauze. Add 50 $\mu \mathrm{L}$ primary antibody on each slide, and incubate slides at $37^{\circ} \mathrm{C}$ for $1 \mathrm{~h}$. Wash slides in PBS, $3 \times 1$ minute, and drain PBS with dry gauze. Add $50 \mu \mathrm{L}$ secondary antibody on each slide, and incubate slides at room temperature for 30 min. Wash slides in PBS, and add $50 \mu \mathrm{L}$ freshly prepared DAB on each slide. All slides were washed with water and counterstained with hematoxylin. Gradient alcohol dehydration and xylene transparent were performed, and all slides were mounted with neutral balata.

\section{Result determination}

10 high powered microscope fields were observed. Brown membrane staining was defined as IGF-IR or SUSD3 positive, and the staining result was assessed with HercepTestscores. The IHC stained specimens were scored from 0 to $3+$. Score 0: No staining is observed, or membrane staining is observed in $<10 \%$ of the tumor cells. Score 1+:A faint/barely perceptible membrane staining is detected in $>10 \%$ of tumor cells. The cells exhibit incomplete membrane staining. Score 2+:A weak to moderate complete membrane staining is observed in $>10 \%$ of tumor cells. Score $3+$ : A strong complete membrane staining is observed in $>10 \%$ of tumor cells. Score 0 specimens were defined as negative, and score $1 \sim 3+$ specimens were defined as positive. $2+$ and $3+$ specimens were defined as high expression.

\section{Statistical analyses}

The data were analyzed with SPSS10.0 statistical software.Statistical analyses were performed by chi-square test and Spearman's rank correlation coefficient.The data were analyzed with SPSS13.0 statistical software.

\section{Results}

Expression of IGF-IR and SUSD3 in breast cancer tissues and adjacent tissues

In 100 breast cancer tissue specimens, the expression rate of IGF-IR is $86.0 \%(86 / 100)$, while the high expression rate is $76.0 \%(76 / 100)$. In adjacent tissue, however, the expression rate is $30 \%$. The differences were statistical significance $(P<0.05)$. The expression rate of SUSD3 in breast cancer tissue is $78.0 \%(78 / 100)$, and the high expression rate is $53.0 \%(53 / 100)$. In adjacent tissue, the expression rate of SUSD3 is $2.0 \%$, which is significantly lower compared with that in breast cancer tissue $(P<0.05)$. Figure 1

The pathological features of breast cancer is associated with the expression of IGF-IR and SUSD3

The expression of IGF-IR and SUSD3 in breast cancer tissue was independent of age, stage, HER-2 expression, or Ki-67 ( $P>0.05)$. The expression rate of IGF-IR and susd3 in infiltrating ductal carcinoma was higher than that in infiltrating lobular carcinoma, the difference is statistically significant $(P<0.05)$. The expression rate of IGF-IR and SUSD3 in ER high expression $(++\sim+++)$ patients was significantly higher than that in ER low expression (- +) patients, and the difference was statistically significant $(P<0$ 05). In 10 cases of triple negative breast cancer patients, IGF-IR and SUSD3 were expresse dpositive lyin

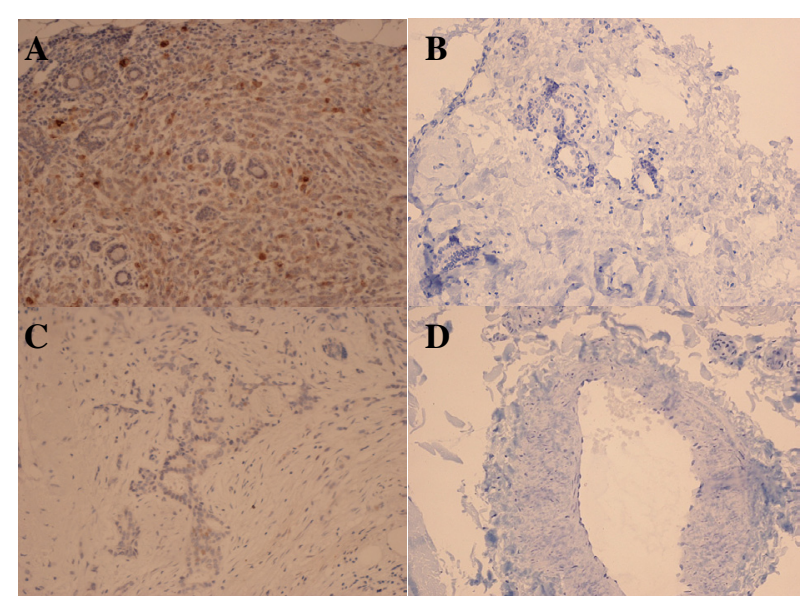

Figure 1. The Expression of IGF-IR and SUSD3 in Infiltrating Ductal Carcinoma and Adjacent Tissues. (Max Vision $\times 200$ )A: Positive expression of IGF-IR in breast cancer tissue; B: Negative expression of IGF-IR in adjacent tissue; C: Positive expression of SUSD3 in breast cancer tissue; D: Negative expression of SUSD3 in adjacent tissue 
DOI:http://dx.doi.org/10.7314/APJCP.2015.16.18.8633 Expression and Clinical Significance of Sushi Domain-containing Protein 3 (SUSD3) and Insulin-like Growth Factor-I Receptor (IGF-IR) in Breast Cancer

Table 1. The Pathological Features of Breast Cancer is Associated with the Expression of IGF-IR and SUSD3

\begin{tabular}{|c|c|c|c|c|c|c|c|c|}
\hline \multirow{2}{*}{\multicolumn{2}{|c|}{ Pathological Features }} & \multirow[t]{2}{*}{$\mathrm{n}$} & \multicolumn{2}{|c|}{ IGF-IR } & \multirow[t]{2}{*}{$P$} & \multicolumn{2}{|c|}{ SUSD3 } & \multirow[t]{2}{*}{$P$} \\
\hline & & & + & - & & + & - & \\
\hline \multirow[t]{2}{*}{ Age } & $>50$ & 42 & 36 & 6 & 0.583 & 34 & 8 & 0.361 \\
\hline & $\leq 50$ & 58 & 50 & 8 & & 44 & 14 & \\
\hline \multirow[t]{4}{*}{ Stage } & I & 18 & 12 & 6 & 0.481 & 10 & 8 & 0.184 \\
\hline & II & 49 & 45 & 4 & & 40 & 9 & \\
\hline & III & 23 & 21 & 2 & & 20 & 3 & \\
\hline & IV & 10 & 8 & 2 & & 8 & 2 & \\
\hline \multicolumn{9}{|c|}{ Pathological Type } \\
\hline \multirow{2}{*}{\multicolumn{2}{|c|}{$\begin{array}{l}\text { invasive ductal carcinoma } \\
\text { invasive lobular carcinoma }\end{array}$}} & 90 & 80 & 10 & 0.032 & 73 & 17 & 0.04 \\
\hline & & 10 & 6 & 4 & & 5 & 5 & \\
\hline \multirow[t]{4}{*}{ ER } & - & 25 & 16 & 9 & 0.0005 & 12 & 13 & 0.004 \\
\hline & + & 25 & 20 & 5 & & 21 & 4 & \\
\hline & ++ & 25 & 25 & 0 & & 22 & 3 & \\
\hline & +++ & 25 & 25 & 0 & & 23 & 2 & \\
\hline \multirow[t]{4}{*}{ HER-2 } & - & 26 & 19 & 7 & 0.621 & 13 & 13 & 0.159 \\
\hline & + & 45 & 42 & 3 & & 40 & 5 & \\
\hline & ++ & 14 & 13 & 1 & & 14 & 0 & \\
\hline & +++ & 15 & 12 & 3 & & 11 & 4 & \\
\hline \multirow[t]{2}{*}{ Ki-67 } & $<50 \%$ & 90 & 78 & 12 & 0.424 & 70 & 20 & 0.553 \\
\hline & $\geq 50 \%$ & 10 & 8 & 2 & & 8 & 2 & \\
\hline
\end{tabular}

Table 2. Correlation between the Expression of IGF-IR and SUSD3 in Breast Cancer

\begin{tabular}{|c|c|c|c|c|c|c|}
\hline \multirow[t]{2}{*}{ SUSD3 } & \multicolumn{4}{|c|}{ IGF-IR } & \multirow[t]{2}{*}{$r$} & \multirow[t]{2}{*}{$P$} \\
\hline & - & + & ++ & +++ & & \\
\hline - & 12 & 7 & 2 & 1 & 0.553 & $<0.01$ \\
\hline+ & 2 & 2 & 10 & 11 & & \\
\hline++ & 0 & 1 & 12 & 13 & & \\
\hline +++ & 0 & 0 & 12 & 15 & & \\
\hline
\end{tabular}

4 cases and 0 cases, respectively. Table 1.

Correlation between the expression of IGF-IR and SUSD3 in breast cancer

In 86 cases of IGF-IR positive patients, SUSD3 was positively expressed in 76 cases. In 14 cases of IGF-IR negative patients, SUSD3 was positively expressed in 2 cases. The expression of IGF-IR was positively correlated with the expression of SUSD3 $(\mathrm{r}=0.553, P<0.01)$. Table 2 .

\section{Discussion}

Compared with other solid tumors, breast cancer has very promising therapeutic prospects.Treatments to breast cancer include surgery, radiotherapy, chemotherapy, endocrine therapy, and targeted therapy.However, breast cancer can lead to a serious of physical and mental impact on patients. As a result, breast cancer prognosis is very important.IGF-IR plays an important role in the development and maintenance of cell malignant phenotype. This present study shows that the positive expression rate of IGF-IR in breast cancer was $86.0 \%$, and the high expression rate of IGF-IR in breast cancer was $76.0 \%$, significantly higher than that in adjacent tissues. And the test results are consistent with the result of radioimmunoassay and immunocytochemistry (ICC) by other researchers (Surmacz, 2000). The present study also found the expression rate of IGF-IR and SUSD3 in ER-positive breast cancer tissue was significantly higher than that in ER-negative tissue.Our previous studies have shown that IGF-IR has interaction with ER in MCF-7 and NWTB3 breast cancer cell lines, and estrogen can induce the expression IGF-IR (Yu et al., 2013a). However, there is no report about association between SUSD3 and IGF-IR currently. This study shows a positive correlation between SUSD3 and IGF-IR.

In this study, among 10 cases of triple negative breast cancer patients, four were IGF-IR positive, and all 10 patients were SUSD3 negative.SUSD3, located in 9q22.3, is a cell surface protein with extracellular, transmembrane and cytoplasmic domains.It is highly expressed in breast cancer and estrogen-sensitive tissues, such as liver, breast, myometrium, endometrial and ovarian. In addition, its secreted extracellular domain can be detected in blood. However, the specific function of SUSD3 remains unknown. Parris et al, identified 12 genes related to invasive breast cancer based on their expression levels and expression patterns in diploid tumors (Parris et al., 2010). They also found these 12 genes significantly associated with the survival of breast cancer patients. Among these 12 genes, 3 genes were highly expressed in invasive breast cancer, while 9 of them were significantly down-regulated. The expression of SUSD3 in invasive malignant breast tumors is particularly low, which is consistent with the results of this study.

In this study, the positive rate of IGF-IR, SUSD3 in stage III and stageIV breast cancer was higher than that in stage I and stage II, but the difference was not statistically significant.A possible explanation was that most of the patients in this study were in early stage. The expression of IGF-IR and SUSD3 were low in triple negative breast cancer, and their positive expression rate in HER-2 positive patients were higher than that in HER-2 negative patients. The expression of IGF-IR and SUSD3 were also higher in $\mathrm{Ki}-67>50 \%$ breast cancer tissue than that in $\mathrm{Ki}-67<50 \%$ breast cancer tissue. But the differences were not statistically significant.Studies have shown that high $\mathrm{Ki}-67$ expression in breast cancer 
Zhao Shuang et al

is an important indicator of prognosis (Casa et al., 2012). And studies have shown that IGF-IR and SUSD3 are also related to cell growth and migration, thus affecting the development of tumors (Bartella et al., 2012; Casa et al., 2012). SUSD3 is a downstream responsive gene of the estrogen receptor. Through genome-wide microarray analysis, researchershave found that SUSD3 is an important mediator of estrogensignal pathways in breast cancer cells, and SUSD3 may also participate in the metastasis of estrogen-dependent breast cancer cells (Moy et al., 2015). Thus, SUSD3 is likely to become an important predictive and prognostic marker of breast cancer after ER and PR.

In this present study, the expression of IGF-IR and SUSD3 was significant higher in invasive ductal carcinoma than that in invasive lobular carcinoma. A possible explanation was that most of the cases in this study were invasive ductal carcinoma. In this study, 97 of all 100 patients were currently alive, and the patients received surgery after 2008. As a result, survival analysis was not performed.

In summary, IGF-IR and SUSD3 may become novel tumor markers which are able to predict the treatment efficacy of breast cancer. These genes are of great importance to prediction of breast cancer prognosis and adjuvant therapy efficiency. Further study on these genes and their signaling pathway may provide new ideas for the treatment and prognosis of breast cancer.

\section{References}

Abbasi S, Rasouli M, Nouri M, et al (2013). Association of estrogen receptor-alpha A908G (K303R) mutation with breast cancer risk. Int J Clin Exp Med, 6, 39-49.

Bartella V, De Marco P, Malaguarnera R, et al (2012). New advances on the functional cross-talk between insulin-like growth factor-I and estrogen signaling in cancer. Cell Signal, 24, 1515-21.

Bulun SE, Chen D, Moy I, et al (2012). Aromatase, breast cancer and obesity: a complex interaction. Trends Endocrinol Metab, 23, 83-9.

Casa AJ, Potter AS, Malik S, et al (2012). Estrogen and insulinlike growth factor-I (IGF-I) independently down-regulate critical repressors of breast cancer growth. Breast Cancer Res Treat, 132, 61-73.

Dabydeen SA, Kang K, Diaz-Cruz ES, et al (2015). Comparison of tamoxifen and letrozole response in mammary preneoplasia of ER and aromatase overexpressing mice defines an immune-associated gene signature linked to tamoxifen resistance. Carcinogenesis, 36, 122-32.

Moy I, Todorovic V, Dubash AD, et al (2015). Estrogendependent sushi domain containing 3 regulates cytoskeleton organization and migration in breast cancer cells. Oncogene, 34, 323-33.

Parris TZ, Danielsson A, Nemes S, et al (2010). Clinical implications of gene dosage and gene expression patterns in diploid breast carcinoma. Clin Cancer Res, 16, 3860-74.

So WK, Leung DY, Ho SS, et al (2013). Associations between social support, prevalent symptoms and health-related quality of life in Chinese women undergoing treatment for breast cancer: a cross-sectional study using structural equation modelling. Eur J Oncol Nurs, 17, 442-8.

Surmacz E (2000). Function of the IGF-I receptor in breast cancer. J Mammary Gland Biol Neoplasia, 5, 95-105.
Yu Z, Gao W, Jiang E, et al (2013a). Interaction between IGFIR and ER induced by E2 and IGF-I. PLoS One, 8, 62642.

Yu Z, Jiang E, Shangguan AJ, et al (2013b). Aromatase in adipose tissue: Relationship to breast cancer. J Med Postgra, 26, 1320-5.

Yu Z, Wang X, Chen L, et al (2013c). Interaction between insulin-like growth factors I receptor and estrogen receptor induced by estradiol and insulin-like growth factors I. J med Postgra, 26, 907-11. 